\title{
LA RELEVANCIA FILOSÓFICA DE LA EDUCACIÓN MUSICAL EN LA POLÍTICA DE ARISTÓTELES
}

\author{
Viviana Suñol* \\ Recebido em: 19/11/2020 \\ Aprovado em: 04/12/2020
}

RESUMEN: El programa de educación musical que Aristóteles presenta en Política VIII y, de manera general, sus reflexiones sobre el empleo de la mousiké en el mejor régimen son considerados como una de las fuentes más importantes para el estudio de la música griega antigua. Sin embargo, son muy escasas las investigaciones que destacan la relevancia filosófica que esta disciplina tiene en el pensamiento aristotélico. Si bien es cierto que el propio Estagirita reconoce no ser un especialista en filosofía de la educación musical e incluso delega el estudio específico de ciertas cuestiones técnicas, no obstante, el papel que le otorga a la mousiké en el diseño del programa educativo ideal y la jerarquía de funciones que le atribuye dan cuenta del destacado lugar que ocupa en su proyecto éticopolítico. Precisamente, el propósito de este artículo es demostrar que para Aristóteles la mousiké no solo es una parte esencial de la vida y de la organización política, sino, más aun, es condición para la vida feliz de los ciudadanos. Con vistas a ello, en la primera parte del trabajo, muestro cómo esta disciplina se inserta en el proyecto del mejor régimen y de la vida mejor y cómo se articula con ambos. En la segunda parte, analizo las distintas funciones que Aristóteles le otorga, su organización jerárquica y la compleja interdependencia que establece entre ellas.

PALABRAS CLAVE: Aristóteles; mousiké; Política; mejor régimen; vida mejor; educación; ocio; kátharsis.

\section{THE PHILOSOPHICAL RELEVANCE OF MUSICAL EDUCATION IN ARISTOTLE'S POLITICS}

*Investigadora

Adjunta del

Consejo Nacional

de Investigaciones

Científicas y

Técnicas, Instituto

de Investigaciones

en Humanidades y

Ciencias Sociales,

Universidad Nacional

de La Plata.

vsunyol@gmail.com

\footnotetext{
ABSTRACT: The musical educational program that Aristotle presents in Politics 8 and more generally his reflections on the use of mousike in the best regime are considered one of the most important sources for the study of Ancient Greek music. However, there are very few studies that
} 
highlight the philosophical relevance that this discipline has to the Aristotelian thought. While it is true that the philosopher himself acknowledges that he is not a specialist in the philosophy of musical education and even delegates the specific study of certain technical issues, nevertheless, the role that he gives to mousike in the design of the ideal educational program and the hierarchy of functions attributed to it give an account of the prominent place that this discipline occupies in his ethico-political project. Precisely, the purpose of this paper is to demonstrate that mousike is not only an essential part of life and the polis, but even more is a condition for the happy life of citizens. In the first part of the work, I show how this discipline is inserted into the political project of the best regime and the best life and how it articulates with both. In the second part, I analyze the different functions that Aristotle gives to it and the complex and hierarchical interdependence that he establishes between them.

KEYWORDS: Aristotle; mousike; Politics; best regime; best life; education; leisure; kátharsis.

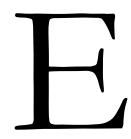
1 programa de educación musical que Aristóteles presenta en Política VIII y, de manera general, sus reflexiones sobre el empleo de la mousiké en el mejor régimen son considerados como una de las fuentes más importantes para el estudio de la música griega antigua (Barker, 1984). ${ }^{1}$ Sin embargo, son muy escasos los estudios que destacan la relevancia que esta disciplina tiene para el filósofo. ${ }^{2}$ En efecto, la mayoría de los intérpretes que se dedican al estudio de la obra aristotélica y de los historiadores de la música omiten las implicancias filosóficas que este tema tiene en su pensamiento. Probablemente, esto se deba al hecho de que sus consideraciones musicales ocupan un lugar marginal en el corpus y están inconclusas. Asimismo, el propio Estagirita reconoce no ser un especialista en el tema e incluso delega el estudio específico de ciertas cuestiones técnicas. Sin embargo, el papel que le otorga a la mousiké en el diseño del programa educativo ideal y la jerarquía de funciones que le atribuye dan cuenta del lugar que esta ocupa en su proyecto ético-político. ${ }^{3}$

\footnotetext{
${ }^{1}$ En el presente artículo resumo los principales resultados de algunos trabajos previos referidos al papel de la mousiké en Pol. VII-VIII. Mi propósito aquí es destacar la importancia de esta disciplina y demostrar las implicancias filosóficas que tiene en el pensamiento ético-político aristotélico. Cf. Suñol (2017, 2018 y 2020).

${ }^{2}$ Aunque existen trabajos que dan cuenta de la importancia de la educación musical (v.gr. Lord, 1982; DePew, 1991; Ford, 2004 etc.), en términos generales, no encuentro que los intérpretes destaquen la articulación del programa de educación musical expuesto en Pol. VIII con el proyecto político aristotélico de Pol. VII. Una excepción es el trabajo reciente de Destrée (2018, p. 183), quien reconoce que esta disciplina es indispensable para el logro de la felicidad perfecta, sin embargo, el autor no ahonda en la correspondencia entre el proyecto político y el currículo musical.

${ }^{3}$ A lo largo del trabajo, mantengo este vocablo en su lengua original con el propósito de resaltar la distancia conceptual que nos separa de la mousiké, puesto que esta era parte esencial de una cultura eminentemente musical y tenía una acepción más amplia que el término actual, próxima a nuestra noción de cultura (Murray; Wilson, 2004, p. 1), pero, sobre todo, era constitutiva de la identidad griega (Rocconi, 2015, p. 82). Además, su empleo en Pol. VIII resulta problemático, véase nota 16.
} 
Precisamente, el propósito de este artículo es demostrar que para Aristóteles la mousiké no solo es parte esencial de la mejor forma de vida en el mejor régimen, sino que es condición para que los hombres alcancen su propósito último: la eudaimonía. Con vistas a ello, en la primera parte del trabajo, muestro cómo esta disciplina se articula e implícitamente se corresponde con la organización política del mejor régimen y el propósito último de la vida mejor. En la segunda parte, analizo las distintas funciones que Aristóteles le otorga, su organización jerárquica y la compleja interdependencia que establece entre ellas.

\section{I}

En primer lugar, es preciso señalar que no es posible comprender la importancia del currículo musical aristotélico si no se tiene en cuenta el desarrollo argumental de los dos últimos libros de la Política, pues el programa de educación musical que Aristóteles diseña en Pol. VIII es una parte esencial de la respuesta que ofrece a las dos preguntas fundamentales que formula al inicio de Pol. VII respecto a cuál es el mejor régimen y cuál es la vida mejor. Ambas cuestiones son para el filósofo conceptualmente inseparables (Pol. 1323a14-17) y se vinculan estrechamente a su reflexión sobre la endaimonía. Justamente para responder a ellas diseña una pólis ideal y establece una serie de condiciones para su constitución referidas al emplazamiento geográfico, el clima, la composición étnica y social de los habitantes, la disposición arquitectónica de los edificios públicos y privados etc. Sin duda, el más importante de todos los factores es la educación, puesto que es fundamental para la construcción y preservación de todo régimen político (Pol. 1337a14-16, EN1094a26-b11), y, además, porque solo a través de ella el hombre puede hacerse virtuoso (Pol. 1332a35-36) y completar su naturaleza (Pol. 1337a1-3). A su vez, la mousiké es la disciplina central del currículo aristotélico no solo porque es a la que más atención le dedica, sino fundamentalmente porque gracias a su función más alta prefigura el fin último del hombre y, por ende, el propósito al que debe apuntar el programa educativo ideal. En definitiva, solo a partir de este recorrido argumental es posible comprender por qué esta disciplina es una pieza clave que articula el pensamiento ético-político de Aristóteles y cómo el programa de educación musical se corresponde prácticamente en cada uno de sus aspectos con la organización política y la vida de los ciudadanos y habitantes de la pólis. En efecto, cada una de las etapas que lo componen, los ritmos, armonías e instrumentos que prescribe para cada actividad y las distintas funciones que cumple en la formación de los ciudadanos están supeditadas al proyecto político aristotélico. Como veremos en la segunda parte, a propósito de las funciones musicales, algunas de estas correspondencias resultan evidentes, mientras que otras no son tan evidentes. A continuación, me ocuparé de estas últimas, ya que entiendo que existe una correspondencia estructural entre, por un lado, la organización política y las etapas de la educación musical, y, por otro, entre el ideal de la vida mejor y el propósito último de la educación musical.

El amplio programa educativo que Aristóteles diseña para los ciudadanos del mejor régimen se inicia antes del nacimiento y, probablemente, se extiende hasta la vejez. ${ }^{4}$ El filósofo

\footnotetext{
${ }^{4}$ Cf. Suñol (2015, p. 60, n. 27).
} 
destaca la importancia que tienen los juegos, las narraciones y los relatos míticos, los cuales son parte esencial de la formación de los niños en el seno del hogar durante los primeros siete años. El currículo musical que bosqueja en Pol. VIII 3, 5-7 forma parte del programa de educación pública que se inicia a partir del segundo septenio. ${ }^{5}$ La conexión más evidente entre la organización del mejor régimen y el programa de educación musical reside en la alternancia generacional que Aristóteles plantea en ambos casos. La sucesión en el ejercicio del poder está determinada por la distinción natural de la edad. En tal sentido, afirma que a los jóvenes les conviene ser gobernados y a los adultos, gobernar (Pol. 1332b35-38). ${ }^{6}$ Este recambio generacional y político garantiza la igualdad y, por ende, la estabilidad del régimen al evitar la sublevación de los gobernados. Además, el propio Aristóteles reconoce que la función subordinada tiene una finalidad pedagógica, puesto que se aprende a gobernar siendo gobernado (Pol. 1277b9-13; 1333a2-3). Esta sucesión generacional en el plano político encuentra su correspondencia en la organización del programa de educación musical, más precisamente, en las prescripciones referidas a la ejecución. El análisis acerca de la ejecución musical es sumamente importante porque muestra cómo el filósofo logra hacer de ella una actividad adecuada para los hombres libres. En Pol. VIII 6 deja en claro que el propósito de la educación musical es convertir a los ciudadanos en buenos jueces; de ahí, la necesidad de que estos participen en la ejecución, pues asegura que es difícil o imposible llegar a ser un juez noble de las obras no habiendo participado (Pol. 1340b23-25). Sin embargo, Aristóteles limita su práctica a la juventud y la prohíbe en la edad adulta (Pol.1340b36-38), ya que reconoce que la práctica profesional de la misma, así como la participación en las competencias, en las que el músico se somete a las exigencias del espectador, producen una degradación intelectual y corporal (Pol. 1341a9-13; b6-18). En virtud de lo cual, sostiene que los ciudadanos no pueden participar de estas. Para impedir que la mousiké torne vulgares a los hombres libres establece no solo un límite en cuanto a la edad de las ejecuciones, sino también determina los tipos de melodías, ritmos e instrumentos que estos pueden aprender durante su juventud (Pol. 1340b41-1341a1). El aprendizaje musical forma parte de la preparación de los ciudadanos, por lo cual está supeditado a las actividades militares y políticas que estos deben ejercer (Pol. 1341a5-8). En suma, Aristóteles propone la sucesión generacional tanto para el ejercicio del poder como para la educación musical, pues, así como los ciudadanos aprenden a gobernar siendo gobernados en su juventud, así también de adultos son capaces de juzgar las melodías y gozar correctamente, gracias al aprendizaje musical que adquirieron cuando eran jóvenes.

Además de esta correlación en el aspecto político, existe una analogía significativa entre la naturaleza peculiar del bios praktikós, ${ }^{7}$ que Aristóteles considera como la mejor forma

\footnotetext{
${ }^{5}$ Si bien Aristóteles toma la división de la educación en septenios, en Pol. VII 17 1336b40-1337a1 la modifica ligeramente al considerar que el proceso natural de la pubertad que se inicia a los 16 años marca el comienzo de la segunda etapa educativa.

${ }^{6}$ Platón, República 412c y Leyes 690a.

${ }^{7} \mathrm{El}$ ideal del bios praktikós ha dado lugar a las más diversas interpretaciones, a punto tal que en la literatura académica no existe acuerdo ni siquiera respecto a cuál es el género de vida que el filósofo defiende. Cf. Suñol (2014).
} 
de vida y la función más elevada que le atribuye a la mousikéen el currículo ideal, ya que tanto la vida más elegible como la función ociosa de la mousiké se caracterizan porque no tienen otro propósito más allá de sí mismas. ${ }^{8} \mathrm{~A}$ mi entender, esta es la correspondencia más estrecha que conecta las preguntas planteadas al inicio de Pol. VII y el programa de educación musical diseñado en Pol. VIII. A pesar de la vaguedad con la que describe el bíos praktikós y de las muchas cuestiones que deja sin resolver su análisis de las formas de vida, es innegable el hecho de que en el régimen ideal todos los ciudadanos están comprometidos con el ejercicio de la vida política, gracias a la sucesión generacional en el poder. A la luz de ello, la vida más elegible no conlleva para Aristóteles el retiro de la vida política, ${ }^{9}$ antes bien presupone el cumplimiento de las responsabilidades cívicas que atañen a todos los ciudadanos. En tal sentido, puede decirse que la vida política es condición para la vida activa y, por ende, para la eudaimonía. ${ }^{10}$ Como veremos en la segunda parte, de igual modo ocurre con el propósito último de la mousiké, ya que su empleo ocioso presupone y requiere del ejercicio de su función ética. En definitiva, el carácter autotélico y contemplativo de la vida activa, la cual presupone el ejercicio de la vida política, se corresponde con la función distintivamente ociosa que Aristóteles le reconoce a la mousiké, la cual requiere del ejercicio previo de su función ética.

\section{II}

Aristóteles establece reiteradamente que son tres las funciones musicales, a saber: el juego en la infancia, que en la adultez se constituye como una forma de descanso luego del trabajo; la educación de los futuros ciudadanos; el ocio de estos en su vida adulta. Si bien delimita con bastante precisión los propósitos de cada una de ellas, ${ }^{11}$ un análisis detenido de los capítulos en los que refiere a las capacidades (dúnamis) de la mousiké (Pol. VIII 3, 5 y 7) revela que hay varias cuestiones problemáticas respecto al lugar que algunas de ellas ocupan en la jerarquía de funciones que el filósofo establece, cuántas son y cómo se vinculan entre

\footnotetext{
${ }^{8}$ En Pol. VII 3 (1325b15-21) Aristóteles aclara que lo práctico no necesariamente es para otros (pròs hetérous) ni los pensamientos prácticos son solo aquellos que tienen en vista lo que resulta a partir de la acción, sino muchos más los que tienen su propio fin (tàs autotelểs) y las contemplaciones y pensamientos que son a causa de sí mismos (bautôn béneken). Cuando en Pol. VIII 3 (1338a10-13) justifica la introducción de la mousiké en el programa educacional por su carácter ocioso, afirma que en el ocio en pasatiempos se aprende algo y se es educado y las cosas que son enseñadas y las que son aprendidas son con vistas a sí mismas (heautôn eînai chárin), mientras que las que son para el trabajo son necesarias y con vistas a otros (chárin állon).

${ }^{9}$ Idea que es ajena al pensamiento griego arcaico y clásico. Cfr. Demont (1990, p. 402-3) y Carter (1986, p. 173).

${ }^{10}$ Suñol (2013, p. 42).

${ }^{11}$ Aristóteles menciona en varias ocasiones la jerarquía de las funciones musicales a lo largo de Pol. VIII. En el capítulo 5 introduce esta enumeración en Pol. 1339a15-26 y, luego, la vuelve a mencionar en Pol. 1339b13-14 con una muy ligera modificación en el orden de las dos primeras funciones. En el último capítulo, en Pol. 1341b36-41, introduce cambios significativos respecto de las formulaciones anteriores. Este último pasaje plantea múltiples dificultades, algunas de las cuales menciono a continuación.
} 
sí. A continuación, presento un resumen de las características de cada una de las funciones y de los principales aspectos problemáticos que comporta su estudio.

\section{El OCIO COMO FIN ÚLTIMO DE LA EDUCACIÓN MUSICAL}

Si bien el Estagirita basa su diseño curricular en el modelo educativo tradicional, la relevancia que le otorga al ocio es un aspecto característico de su pensamiento. ${ }^{12}$ Este empleo de la disciplina musical es el más importante, por cuanto se vincula al fin último al que apunta la educación y, de manera general, la vida de los hombres libres, esto es, la endaimonía. ${ }^{13}$ Precisamente, la educación musical introduce a los futuros ciudadanos en el ejercicio de una actividad no instrumental que no tiene otro propósito más allá de sí misma. El ocio como actividad propia de los hombres libres no refiere a la inactividad, sino por el contrario a la forma más elevada del hacer, cuyo modelo metafísico sería el del Motor inmóvil, y que, en el plano humano, se correspondería con la actividad contemplativa propia de la filosofía. ${ }^{14}$ Como vimos en la primera parte, este modelo autotélico y reflexivo de actividad que caracteriza a la singular definición de la vida práctica como forma de vida más elegible en Pol. VII 3 (1325b16-23), se corresponde en el programa educativo aristotélico con el empleo ocioso de la mousiké como la forma primaria en la que los ciudadanos ejercitan el ocio (Pol. 1338a32-34).

A diferencia de las restantes disciplinas que conforman el programa de educación pública, la mousiké es la única cuya presencia en el currículo justifica, probablemente porque se trata de un pasatiempo en el ocio (Pol.1338a21-22) y, como tal, carece de un propósito o utilidad definidos (Pol. 1337b27-28). Al señalar que este empleo ocioso solo está reservado para los hombres libres, Aristóteles lo distingue de la mera relajación a la que da lugar su utilización luego del trabajo y que es propia del estamento social más bajo, mientras que la función ociosa es exclusiva de los ciudadanos. Este modelo educativo centrado en el ocio presupone que los trabajadores, que están excluidos de la ciudadanía, no pueden practicar el ocio y, en consecuencia, tampoco pueden aspirar a la eudaimonía. La noción misma de ocio se sustenta en prejuicios aristocráticos que determinan el desprecio aristotélico por las actividades manuales, lo cual se manifiesta -como vimos en la primera parte- en las restricciones que establece sobre la ejecución musical en la vida adulta, ya que esta actividad artesanal puede degradarlos corporalmente, afectando su desempeño cívico. En definitiva, la diferencia fundamental que Aristóteles establece entre el ocio musical propio

\footnotetext{
${ }^{12} \mathrm{El}$ ocio adquiere una singular relevancia en el pensamiento aristotélico, cuyas reflexiones tienen un carácter fundacional, gracias a la conceptualización y articulación del tema en su obra. Para un análisis detallado y, en algunos aspectos, contrastante del tema, cf. Nightingale (1996, p. 29-39); Solmsen (1964); Lord (1982) y Demont (1990).

${ }^{13}$ Para un resumen de la amplia discusión académica sobre la endaimonía, véanse Suñol (2013 y 2014, p. 298-302).

${ }^{14}$ Sobre la problemática alusión a la philosophía como paradigma de la actividad contemplativa en Pol. VII, cfr. Suñol (2014, p. 312-15).
} 
de los ciudadanos adultos y el empleo meramente relajante de esta disciplina por parte de la mayoría de los habitantes, está determinada no solo por la organización social, política y económica del mejor régimen, sino también por la valoración filosófica suprema de las actividades no instrumentales y el desprecio por las que tienen un carácter necesario y sirven a un propósito externo.

La mousiké constituye el modelo de la educación liberal y bella, cuyo objeto son las cosas que se realizan por sí mismas (Pol. 1338a9-12, 30-32). Aunque Aristóteles no especifica el objeto y el tipo de aprendizaje al que da lugar esta función, los indicios que ofrece sugieren que el objeto del ocio musical son las bellas melodías y armonías o, quizás incluso, los bellos espectáculos teatrales (Pol. 1338b1-2), y, que, gracias a ella, los ciudadanos aprenden a ser buenos oyentes y espectadores, es decir, adquieren la capacidad de juzgar la belleza. ${ }^{15}$ Esta actividad no tiene otro propósito más que la contemplación y/o escucha de la bella mousiké y, como tal, conlleva la forma más elevada del placer. Precisamente, este carácter contemplativo es el que emparienta el ocio musical con la eudaimonía. En consecuencia, el empleo ocioso de la mousiké excluye toda forma de ejecución y solo refiere a la figura del oyente y/o espectador, dependiendo de la amplitud semántica que se le reconozca a este término. ${ }^{16}$

En contraste con la importancia que Aristóteles le otorga a la función ociosa en Pol. VIII 3 y con la escueta mención en Pol. VIII 5 a su contribución al discernimiento (phrónesis), ${ }^{17}$ en el final del tratado parece identificarla con el descanso y la relajación de las tensiones, al reunirlas como parte de la tercera función que enuncia en el pasaje (Pol. 1341b 40-41), lo cual contradice no solo los cimientos del programa de educación musical, sino también los principios sobre los que se fundamenta su proyecto ético-político. ${ }^{18}$ Lejos de ser un aspecto secundario, esta confusa y errónea noción del ocio entendida como mera relajación pone en tela de juicio la comprensión misma de lo que Aristóteles entiende por el propósito último de la vida mejor. A mi juicio, las dificultades que presenta el pasaje en cuestión no

\footnotetext{
${ }^{15}$ Es sugestivo que en las referencias a esta función emplee kalós (Pol. 1339b18, 1340b38), mientras que para aludir a la función ética utilice chrestá (Pol. 1339b3), lo cual sugiere que el juicio al que da lugar la función ociosa refiere a la belleza, mientras que en el caso de la función ética se trata de un juicio referido a la utilidad práctica.

${ }^{16}$ Aristóteles no aclara qué entiende por mousiké en Pol. VIII 3, 5-7. Un relevamiento de sus empleos sugiere que, de manera general, adopta una comprensión amplia, la cual se manifiesta en su expresa alusión tanto a la música instrumental como con cantos (1339b20-21), además su clasificación de los tipos de espectadores (1342a19-21), revela que también refiere al teatro. Sin embargo, cuando analiza la influencia ética de la mousiké en Pol. VIII 5, parece centrarse en los componentes instrumentales de esta disciplina. Para lecturas contrapuestas sobre esta cuestión, cfr. Lord (1982, p. 29 y 34); Ford (2004, p. 309 y 315); Halliwell (2002, p. 244).

${ }^{17}$ En Pol. VIII 51339 a25-26 refiere a la función ociosa afirmando que contribuye en algo al pasatiempo y a la phrónesis. Aunque algunos intérpretes traducen este empleo de la palabra en términos de sabiduría práctica, parece más apropiado entenderla en su sentido intelectual, esto es, refiriendo al tipo de discernimiento al que da lugar el ocio.

${ }^{18}$ Aristóteles expone estos principios en Pol. VII 14. La división del alma y de la vida que allí propone guarda una íntima conexión con la importancia que le otorga a la función ociosa de la mousiké.
} 
se resuelven únicamente con la eliminación de esta referencia al ocio (pròs diagogén), a la que algunos autores de manera plausible consideran una glosa, pues este legítimamente forma parte del esquema tripartito de funciones. ${ }^{19}$ En realidad, es la kátharsis la que no cuadra en esta enumeración de funciones y, justamente, es su presencia la que debe ser explicada. ${ }^{20}$

Una de las mayores dificultades que se plantea en torno a la función ociosa reside en el hecho de que Aristóteles nunca aclara cuáles son las melodías, armonías e instrumentos que le corresponden. La triple división de las melodías en éticas, prácticas y entusiásticas que enuncia en Pol. VIII 7 (1341b32-34) no permite esclarecer esta cuestión con certeza, pues solo las melodías éticas guardan correspondencia con la función educativa, mientras que respecto de las prácticas y las entusiásticas, se limita a señalar que están destinadas a los oyentes, es decir, no son para ser ejecutadas. El principal obstáculo de esta división reside en las melodías prácticas, las cuales tradicionalmente eran vinculadas a la actividad guerrera, pero cuyo significado en el pasaje resulta muy difícil de elucidar. ${ }^{21}$ Existe un amplio debate en la literatura académica en torno de esta cuestión, de hecho, podría decirse que existen tantas respuestas como intérpretes del texto. ${ }^{22}$ Lo cierto es que Aristóteles reconoce que esta división de las melodías no es propia y que la toma de otros filósofos y músicos dedicados al estudio de la educación musical. En varios pasajes remite a estos especialistas para un estudio detallado de estas cuestiones, lo que demuestra la importancia que el tema tenía para los pensadores y músicos de la época. ${ }^{23}$ Teniendo en cuenta el origen heredado de esta distinción que Aristóteles efectivamente adopta, el carácter esquemático de su análisis, las dificultades textuales del pasaje y, sobre todo, la re-sistematización de la noción de ocio y de la teoría musical a la luz de su propio proyecto filosófico, no es posible dar una respuesta concluyente sobre cuáles son las melodías que corresponden a la función ociosa.

\footnotetext{
${ }^{19}$ Destrée (2017, p. 37) propone eliminar esta referencia al ocio, a la que considera como una glosa, lo que - a su entender - permite destacar la originalidad de la comprensión aristotélica de la mousiké. En general, todo el pasaje parece no corresponderse con las formulaciones previas de los empleos de la disciplina musical, lo cual ha motivado a proponer diversos cambios o enmiendas textuales. Cf. Schütrumpf (2005, p. 651-2).

${ }^{20}$ Cuestión de la que me ocupo en la última sección.

${ }^{21}$ Para algunos intérpretes estas refieren a la función ociosa, mientras que otros las identifican con el descanso de las tensiones del trabajo propio de la clase más baja de espectadores. Para un relevamiento de las distintas interpretaciones propuestas, cf. Schütrumpf (2005, p. 669).

${ }^{22}$ Por ejemplo, Destrée (2013, p. 13) propone identificar fácticamente la función ociosa con la función ética, de modo que las melodías éticas coincidirían con las ociosas y lo único que diferenciaría es quiénes las utilizan en cada caso y con qué finalidad.

${ }^{23}$ Aristóteles alude a estos especialistas en varios pasajes y los divide en dos grupos: los filósofos dedicados a la educación musical y los expertos en música (Pol. 1340b6, b18, b34, 27-28; 1341 b33 y 1342b8-9). Los intérpretes suelen considerar que mediante estas alusiones refiere a sus discípulos: Aristoxeno, Teofrasto y Heráclides Póntico. Sin embargo, Barker (2005, p. 100) advierte que cualquier intento de identificar las fuentes de Aristóteles es incierto, ya que se trataba de una cuestión ampliamente debatida y que involucraba a intelectuales de diversos orígenes.
} 


\section{LA EDUCACIÓN MUSICAL COMO FORMACIÓN ÉTICA DE LOS FUTUROS CIUDADANOS}

La mousiké cumple una función ética fundamental, debido a que, como parte de un programa educativo integral, contribuye a formar el carácter de los futuros ciudadanos, gracias a la influencia que ejerce en el carácter del alma mediante las emociones que despierta en los oyentes. La prueba que Aristóteles ofrece para demostrar esta influencia sobre el carácter del alma es el efecto entusiástico que producen las melodías de Olimpo, lo cual resulta muy significativo, ya que recurre a una experiencia emocional extrema, que no forma parte del programa educativo musical, pero que quizás, por su carácter extático es la que mejor ilustra dicha influencia. ${ }^{24}$ Tanto para demostrar el influjo ético de la mousiké como para dar cuenta del carácter representacional de los ritmos y las melodías, apela a los "hechos", esto es, los cambios emocionales que esta produce en los oyentes. ${ }^{25} \mathrm{El}$ poder simpatético ${ }^{26}$ que le atribuye a la mousiké se sustenta en el supuesto, por entonces ampliamente difundido, de que en las melodías y ritmos existen semejanzas con la verdadera naturaleza de los caracteres (Pol. 1340a39, 18-19). ${ }^{27}$ Si bien Aristóteles deja en claro que además de esta, toma otras ideas y conceptos que formaban parte del amplio debate que en aquellos tiempos existía en torno a esta disciplina, lo cierto es que reinterpreta algunas de estas nociones y las integra como parte de su propio pensamiento. ${ }^{28}$ En este sentido, establece una estrecha correspondencia entre su definición de la virtud ética y el goce musical, de modo que la educación musical funciona a la vez, como un proceso de habituación o entrenamiento ético. ${ }^{29}$ Más allá de

\footnotetext{
${ }^{24} \mathrm{La}$ importancia de la referencia a estas melodías no solo reside en el hecho de que sugiere un vínculo con la función catártica (cuestión de la cual me ocuparé en la sección correspondiente), sino también por el origen frigio de la figura legendaria de Olimpo y su carácter de fundador de la aulética. Como veremos más adelante, las referencias etno-musicales resultan claves para entender el programa educativo musical aristotélico. Sobre la figura de Olimpo, cf. Kraut (1997, p. 193); Barker (1984, p. 92). ${ }^{25}$ Pol.1340a12-13, 21-23; a39-b10, especialmente b6-7.

${ }^{26}$ En Pol. 1340a13 Aristóteles refiere al carácter simpatético de la mousiké y pareciera aludir a los discursos, excluyendo a las melodías y ritmos. Este es un pasaje decisivo porque pone en tela de juicio las numerosas lecturas que aducen que Aristóteles emplea aquí un sentido estrecho de mousiké. Para evitar esta dificultad muchos autores siguen la enmienda propuesta por Susemihl. Al respecto, cf. Ford (2004, p. 320-1, n. 35).

${ }^{27}$ Como es bien sabido, esta idea estaba ampliamente difundida entre los griegos, era el eje de la teoría musical damoniana y, posteriormente, de la concepción musical platónica. Sin embargo, existen testimonios de que la denominada teoría del êthos musical no era universalmente compartida. Cf. Barker (2005, p. 105). Sobre el origen de esta creencia en la medicina hipocrática y la filosofía presocrática, cf. Woerther (2008, p. 93-4; 2002, p. 36 y 2007, p. 181).

${ }^{28}$ Según Cagnoli Fiecconi (2016), Aristóteles no se limita a aceptar de manera no reflexiva esta creencia tradicional, sino que provee una fundamentación filosófica sólida, gracias a las similitudes que establece entre los aspectos propiamente progresivos de la mousikée, esto es, melodía y ritmo, con el desarrollo temporal, belleza y propósitos de las acciones y caracteres.

${ }^{29}$ Varios trabajos recientes destacan, en mayor o menor medida, el vínculo que la educación musical tiene con el pensamiento ético de Aristóteles. Cf. Ford (2004, p. 316 y 332); Hitz (2012, p. 270);
} 
la complejidad que subyace a la sucinta presentación del tema ${ }^{30}$ Aristóteles fundamenta dicha correspondencia en dos razones: por un lado, en la proximidad que existe entre el sufrimiento y el goce que experimentamos ante las semejanzas y lo que sentimos ante lo que efectivamente ocurre (Pol. 1340a25-28), ${ }^{31}$ lo cual no solo atañe a la mousiké, $;{ }^{32}$ por otro lado, la singularidad de la percepción auditiva, en la medida en que solo ella permite establecer una semejanza directa con los caracteres y es universal, lo que se contrapone al carácter mediato y limitado de los signos que caracterizan a las artes visuales. ${ }^{33}$ Asimismo, como en todas las funciones musicales, el placer es el origen de la misma, si bien se trata de una forma superior al placer musical "común" que -como veremos a continuación-caracteriza a la función más elemental. En cambio, esta es una forma "correcta" o, más precisamente, virtuosa del placer que surge del disfrute de los buenos caracteres y de las acciones bellas (Pol. 1339a24-25).

En definitiva, la naturaleza a la vez ética, simpatética y placentera de la mousiké, hacen de ella un instrumento pedagógico fundamental en la educación de los futuros ciudadanos del mejor régimen. Esto explica la importancia que para el Estagirita tiene determinar el grado de participación de los ciudadanos en la ejecución, así como los instrumentos, las armonías y los ritmos que deben intervenir en la educación musical de los jóvenes y cuáles deben ser excluidos. El hecho de que para la educación solo permita el empleo de la armonía y la melodía doria refleja que esta apunta al equilibrio emocional de los jóvenes, el cual es acorde al carácter estable y valiente que deben tener los ciudadanos del mejor régimen (Pol. 1340b34; 1342a28-30; 1342b12-16). De igual modo, la exclusión del aulós, entre los instrumentos, y del frigio, entre las armonías, debido a su carácter orgiástico y patético, y, probablemente, del ditirambo, en la poesía, revela que el programa de educación musical apunta a controlar los excesos emocionales (Pol. 1341a18-b8; 1340b5; 1342a32-b12)..$^{34}$ Las referencias etnomusicales que pueden identificarse en Política VIII 5-7 ponen de manifiesto que el propósito de la propuesta pedagógica musical aristotélica es el control de las emociones, ${ }^{35}$ el cual permite que por medio de la mousiké los futuros ciudadanos cultiven la moderación durante la infancia y solo sean capaces de experimentar excesos emocionales en la adultez.

Brüllman (2013, p. 368-72); Cagnoli Fiecconi (2016, p. 409; p. 420-2). Para un resumen de estas lecturas, véase Suñol (2020, p. 129, n. 31).

${ }^{30}$ Aristóteles presenta al poder afectivo de la mousiké, su carácter representacional y su función pedagógica como aspectos de un mismo fenómeno que están íntimamente vinculados entre sí, pero no se explican recíprocamente. Sobre las distintas interpretaciones de esta cuestión, cf. Cagnoli Fiecconi (2016, p. 412, n. 8).

${ }^{31}$ Poética 1448b10-19 y Retórica 1371a31-b12.

${ }^{32}$ Para una perspectiva contrapuesta sobre la naturaleza de las emociones que suscita la mousiké, cf. Konstan (2006, p. 39) y Nussbaum (2001, p. 288).

${ }^{33}$ Cf. Halliwell (2002, p. 245-6).

${ }^{34}$ Aristóteles también excluye de su programa musical otros instrumentos como la cítara y, en general, todos los instrumentos que requieren habilidad técnica para su ejecución.

${ }^{35}$ Cf. Rocconi (2010, p. 43). 
Si bien es cierto que la mousiké no conduce por sí sola a la virtud, el ciudadano del mejor régimen no puede alcanzar la virtud ética si no ha sido previamente habituado a ella mediante esta disciplina. El proceso de habituación musical es condición para el desarrollo de la capacidad de discernimiento ético. En otros términos, la función educativa de la mousiké no basta para que los futuros ciudadanos se conviertan en virtuosos, pero es una instancia necesaria de su formación para que aprendan a serlo. ${ }^{36}$

\section{LA FUNCIÓN PRODUCTIVA DE LA MOUSIKÉ, LA FORMA MÁS ELEMENTAL DEL PLACER MUSICAL}

La función más elemental de la mousiké es aquella que corresponde a su empleo como descanso del trabajo y, por ende, esta función está destinada a los trabajadores. Tal como revela la palabra griega que lo designa (ascholía), el trabajo se define como ausencia del ocio e implica fatiga y tensión; razón por la cual, Aristóteles asegura que deben emplearse los juegos para aliviarlos y dar lugar al descanso (anápausis). Si bien en un comienzo alude el papel que los juegos tienen en la infancia, luego en la vida adulta esta función refiere específicamente a su empleo como descanso del trabajo. En virtud de lo cual, he optado por denominarla como función productiva. El placer que produce esta función es fruto de ese movimiento del alma que es la relajación (ánesis). De hecho, en Pol. VIII 5 1339a16-21 la incluye en esta categoría junto con el sueño, la bebida y la danza, describiéndolos como aquellas actividades que hacen cesar las preocupaciones. Precisamente, se trata de la forma más baja del placer musical porque su propósito es aliviar al estamento social inferior (Pol. 1339b26-40; 1342a20-22).

A lo largo de Pol. VIII, Aristóteles destaca la raigambre antropológica del placer musical; de ahí, su reiterada referencia al "placer común" del que todos los hombres de todas las edades y caracteres tienen sensación, que afecta incluso a otros animales, esclavos y niños (Pol. 1339b20-22; 1340a2-5; 1341a15-18). El placer acompaña a todas y cada una de las funciones musicales (Pol. 1342a25), en razón de ello es posible establecer una tácita jerarquía conforme a la cual, el placer concomitante al ejercicio de la función ociosa constituye su forma más elevada, por cuanto surge del disfrute de las bellas melodías y armonías sin ninguna otra finalidad (Pol. 1339b17-19, b25). En el siguiente nivel de esta escala descendiente se sitúa el placer ético de la mousiké, que - como señalamos en el apartado anterior - es la forma del placer musical que permite que esta disciplina sea un instrumento pedagógico privilegiado en la habituación ética de los jóvenes y que se sustenta en el goce de las bellas melodías, cuyo propósito es aprender a discernir los buenos caracteres y las bellas acciones (Pol. 1340a14-18; b15-19). Por último, la forma más elemental del placer musical refiere a su empleo productivo, el cual - como vimos - corresponde al descanso de las tensiones del trabajo. ${ }^{37}$ Las dos formas superiores del placer son exclusivas de los ciudadanos del mejor

\footnotetext{
${ }^{36}$ Cagnoli Fiecconi (2016, p. 422).

${ }^{37}$ Aunque Aristóteles no incluye expresamente a la kátharsis en la triple jerarquía de las funciones musicales, no obstante, le atribuye una forma específica de placer (Pol. 1342a14-15).
} 
régimen. Aunque estrictamente los ciudadanos no trabajan ni pueden dedicarse a actividades artesanales, no están exentos de experimentar las tensiones que, de manera necesaria, conlleva la vida cívica, y, por ende, es muy probable que el filósofo admitiera la posibilidad de que estos eventualmente emplearan la mousiké con vistas a la relajación (Pol. 1337b33-1338a1). ${ }^{38}$

\section{EL LUGAR DE LA KátHARSIS EN LA JERARQUía DE LAS FUNCIONES MUSICALES}

La kátharsis no forma parte de la jerarquía de las funciones musicales, sin embargo, en el último capítulo del tratado (Pol. 1341b36-41) Aristóteles presenta una enumeración que se contrapone en varios aspectos con la división tripartita inicial y en la que refiere a ella como si se tratara de una función diferenciada. ${ }^{39}$ Las reiteradas alusiones al número tres (incluso en este mismo pasaje) sugieren que el Estagirita adopta, al menos de manera expresa, un esquema tripartito, ${ }^{40}$ de modo que no parece plausible pensar en una cuarta función. ${ }^{41}$ Se trata de un tema que aún hoy es objeto de discusión, tal como lo demuestra la reciente publicación del extenso artículo de Ferrari (2019). Tradicionalmente se han adoptado dos líneas de lectura: por un lado, quienes consideran que es "obvia" su identificación con la relajación ${ }^{42}$ y, por otro, quienes vinculan a la kátharsis con la educación, incluso hay quienes postulan una oscilación entre ambas funciones. ${ }^{43} \mathrm{~A}$ decir verdad, un análisis minucioso del tema revela que existen indicios que abonan cada una de estas lecturas. Por razones de extensión no puedo hacer un análisis detenido y debo limitarme a hacer una presentación esquemática.

En primer lugar, es preciso señalar que el papel que desempeña la kátharsis en el esquema de las funciones musicales es difícil de precisar, ya que no resulta convincente la propuesta de identificarla con la mera relajación que caracteriza a la función productiva ni es posible vincularla a la educación ni tampoco la de afirmar su independencia como función musical. Aunque su efecto relajante y la comparación con la cura médica sugieren una conexión con la primera, la relajación que produce y el placer que conlleva no son de la misma clase que la relajación y el placer que se experimenta después de un trabajo duro.

\footnotetext{
${ }^{38}$ La referencia al empleo de las melodías relajadas en la vejez sugiere que en la vida adulta también puede emplearse la mousiké con otros propósitos además del ocio (Pol. 1342b23-24). Cf. Destrée (2011, p. 22).

39 "Decimos que es preciso emplear la mousiké no con vistas a una sola utilidad, sino para muchas (no solo en virtud de la educación y de la kathárseos - a qué llamamos kátharsin, ahora [lo diremos] de manera general (haplôs), pero hablaremos de nuevo más claramente en los [escritos] acerca de la poética - tercero (tríton), para el pasatiempo, para la relajación y para el descanso de las tensiones." La traducción es mía.

${ }^{40}$ Aristóteles emplea tríton en Pol. 1339a25; triôn en Pol. 1339b10 y tríton en Pol. 1341 b40.

${ }^{41}$ Kraut (1997, p. 209), Barker (2005, p. 100) y Destrée (2011, p. 21) hablan de una cuarta función. En su último trabajo, Destrée (2017, p. 40) adhiere al esquema tripartito.

${ }^{42}$ Cf. Destrée (2011, p. 21; 2017, p. 38-40).

${ }^{43}$ Suñol (2018).
} 
En consecuencia, la identificación de la kátharsis con la relajación no resulta tan obvia como algunos autores afirman (pace Destrée 2011, p. 21), lo cual se pone en evidencia en el pasaje mismo de Pol. VIII 7, donde las presenta con funciones diferenciadas. Respecto a su posible vínculo con la función educativa, aun cuando resulta llamativo que apele a las melodías de Olimpo, que tienen un efecto extático, para ejemplificar su influencia ética, Aristóteles deja en claro que la kátharsis no educa moralmente a los futuros ciudadanos. Si bien todas las experiencias emocionales involucran una cierta clase de kátharsis (Pol. 1342a 14), ${ }^{44}$ es probable que sus formas más extremas solo estén permitidas durante la adultez, cuando estos ya han adquirido la virtud. Las emociones y, en particular, los desbordes emocionales -ya sea en ámbitos artísticos y/o cultuales- son parte de la vida de todos los hombres, incluso de los ciudadanos que han alcanzado la moderación y son capaces de disfrutar del ocio musical. En consecuencia, el éxtasis emocional y la posterior relajación que, de manera general, la kátharsis produce, no forman parte del proceso de habituación a la virtud, pero lo presuponen. Precisamente, en este sentido es legítimo afirmar que la función ética de la mousiké es condición de la catártica. En este punto del análisis, la kátharsis pareciera estar en una posición oscilante entre la relajación y la educación, ${ }^{45}$ pero sin poder ser identificada con ninguna de las dos. ${ }^{46}$ Sin embargo, la reciente interpretación propuesta por Ferrari (2019) ofrece una mirada novedosa, ya que es el primer intento de vincular a la kátharsis con el ocio. A su entender, este concepto pertenece al reino conceptual de la diagogé, esto es, el ocio y su patrón o esquema emocional se sustenta en el hecho de que la emoción no es simplemente descargada como ocurre en el caso del entretenimiento propio de los trabajadores, sino descargada por el proceso mismo de ser estimulada y exacerbada. ${ }^{47}$ El autor lo describe como un proceso homeopático en el sentido de que la emoción es producida por la irritación, llevada al clímax y luego es aliviada; ella se construye en el curso de un largo proceso. ${ }^{48}$ La música catártica, categoría que Ferrari redefine al incluir en ella las armonías entusiasticas y prácticas, alivia la agitación que la música misma ha inducido. A diferencia de la relajación que produce la música destinada a los espectadores vulgares, en los que la tensión precede a su alivio, en este caso, el sentimiento solo se produce y es desarrollado por la experiencia musical misma, la cual requiere a la vez de una combinación de un estímulo activo y una sensibilidad receptiva por parte del espectador. El placer que involucra no tiene un sesgo utilitario, sino que es una forma pura del placer que se obtiene en la música misma sin ningún otro fin ulterior. ${ }^{49}$ A partir de lo cual, Ferrari habla de una "superioridad estética" de los hombres libres y educados para la música y sostiene que el placer que la kátharsis pone en juego en los espectadores educados no es de carácter moral

\footnotetext{
${ }^{44}$ Pace Ferrari (2019, p. 132, n. 31).

${ }^{45}$ Cf. Destrée (2017, p. 38).

${ }^{46}$ Suñol (2018, p. 135-6).

${ }^{47}$ Ferrari (2019, p. 125).

${ }^{48}$ Ferrari (2019, p. 137).

${ }^{49}$ Ferrari (2019, p. 141-2).
} 
ni educacional ni es intelectualmente elevado, sino que se trata de un compromiso estético. ${ }^{50}$ Más allá del supuesto esteticista sobre el que se sustenta su lectura y de la singularidad de su interpretación, el reconocimiento del patrón homeopático o, más precisamente podría decirse, reflexivo de la kátharsis, resulta muy valioso por el énfasis que el autor hace en la distinción del placer propio del entretenimiento de los trabajadores y el placer puro del ocio, la cual pone en tela de juicio la "obvia" identificación entre ambos. Recordemos que lo distintivo de la singular y novedosa comprensión aristotélica del ocio reside en su carácter no instrumental y, precisamente, es este aspecto el que permite vislumbrar una posible conexión entre ambas. Sin embargo, la superioridad intelectual que Aristóteles le otorga al ocio se desdibuja en la lectura propuesta por Ferrari.

En conclusión, la kátharsis no es plenamente identificable con ninguna de las tres funciones musicales, pero comparte características con todas ellas. En efecto, produce relajación, pero de un tipo diferente al descanso del trabajo; no forma parte de la educación de los jóvenes, pero presupone un alto grado de conocimiento moral por eso está reservada a los adultos; no involucra un discernimiento intelectual, pero tiene un carácter no instrumental que es propio del ocio.

\section{Conclusiones}

La jerarquía de las funciones musicales que Aristóteles presenta en Pol. VIII se vincula estrechamente al mejor régimen político que bosqueja en Pol. VII, en la medida en que cada una de ellas se corresponde con una determinada edad, grupo social, responsabilidad cívica y tipo de actividad de los habitantes y ciudadanos del mejor régimen. De ahí que no sea posible entender dicha jerarquía si no es a la luz de su proyecto ético-político, y, a su vez, cada una de estas funciones resulta clave para entender en qué consiste la vida mejor en el mejor régimen. Así la función ociosa de la mousiké prefigura el ideal aristotélico de la vida más elegible, pues hemos visto que las dos tienen un carácter contemplativo, autotélico, solo pueden ejercerlas ciudadanos adultos y conducen al fin último de la existencia. Asimismo, significativamente ambas presuponen el ejercicio de la virtud: la función educativa, en el caso del ocio musical, y, la vida política, en el de la vida más elegible. De modo que la función ociosa no solo no excluye a las demás, sino que incluso las presupone. Más aún, independientemente de la problemática ubicación de la kátharsis en la jerarquía de funciones musicales, también esta requiere que quienes, en mayor o menor grado, la experimenten hayan conquistado la moderación emocional gracias al aprendizaje musical adquirido en la juventud. Dicho, en otros términos, la kátharsis supone y requiere el ejercicio previo de la función ética. Además, el placer es el sustrato común, concomitante a todas las funciones, cuya jerarquía también se correlaciona con el esquema tripartito. Esta interdependencia revela que a pesar de la innegable supremacía de su propósito ocioso, las restantes funciones de la mousiké son indispensables no solo para alcanzar el fin último de esta disciplina, sino también

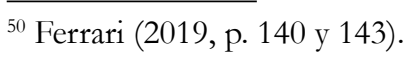


para el logro de la vida mejor en el mejor régimen, dado que no es posible vivir la vida más elegible ni tampoco construir el mejor régimen político, si la educación de los ciudadanos no comprende el ejercicio de las distintas funciones musicales. En conclusión, mediante la extensa y compleja argumentación que elabora en Pol. VII-VIII, Aristóteles deja en claro que la mousiké es condición para alcanzar la vida mejor en el mejor régimen.

\section{REFERENCIAS}

BARKER, Andrew (ed.). Greek musical writings. Cambridge: Cambridge University Press, 1984. v. 1.

BARKER, Andrew. Psicomusicologia nella Grecia Antica. Napoli: Guida Editore, 2005.

BRÜLLMANN, Philipp. Music builds character Aristotle, Politics VIII 5, 1340a14-b5. Apeiron, v. 46, n. 4, p. 345-373, 2013.

CAGNOLI FIECCONI, Elena. Harmonia, melos and rbytmos. Aristotle on Musical education. Ancient Philosophy, v. 36, n. 2, p. 409-424, 2016.

CARTER, Lynn. The quiet Athenian. Oxford: Clarendon Press, 1986.

DEMONT, Paul. La cité grecque archä̈que et classique et l'idéal de la tranquillité. Paris: Belles Lettres, 1990.

DEPEW, David. Politics, music and contemplation in Aristotle's Ideal State. In KEYT, David; MILLER, Fred (ed.). A companion to Aristotle's Politics. Oxford: Blackwell, 1991, p. 346-380.

DESTRÉE, Pierre. Aristotle and musicologists on three functions of music. A note on Pol. 8, 1341b401. Greek and Roman Musical Studies, v. 5, p. 35-42, 2017.

DESTRÉE, Pierre. Aristotle on music for leisure. In D’ANGOUR, Armand; PHILLIPS, Thomas (ed.). Music, texts, and culture in Ancient Greece. Oxford: Oxford University Press, 2018, p. 183-202.

DESTRÉE, Pierre. Education, leisure, and politics. In DESLAURIERS, Marguerite; DESTRÉE, Pierre (ed.). The Cambridge companion to Aristotle's Politics. Cambridge: Cambridge University Press, 2013, p. 301-323.

DESTRÉE, Pierre. La purgation des interprétations: conditions et enjeux de la catharsis poétique chez Aristote. In DARMON, Jean-Charles (ed.). Littérature et thérapentique des passions: la catharsis en question. Paris: Hermann, 2011, p. 14-35.

FERRARI, John. Aristotle on musical catharsis and the pleasure of a good story. Phronesis, v. 64, p. 117-171, 2019.

FORD, Andrew. Catharsis. The Power of Music in Aristotle's Politics. In MURRAY, Penelope; WILSON, Peter (ed.). Music and the Muses. Oxford: Oxford University Press, 2004, p. 309-336. 
HALLIWELL, Stephen. The aesthetics of mimesis. Ancient texts and modern problems. Princeton: Princeton University Press, 2002.

HITZ, Zena. Aristotle on law and moral education. Oxford Studies in Ancient Philosophy, v. 42, p. 263-306, 2012.

KONSTAN, David. The emotions of the Ancients Greeks. Toronto: University of Toronto Press, 2006.

KRAUT, Richard. Aristotle Politics Books VII and VIII. Oxford: Oxford University Press, 1997.

LORD, Carnes. Education and culture in the political thought of Aristotle. New York: Cornell University Press, 1982.

MURRAY, Penelope; WILSON, Peter (ed.). Music and the Muses. The culture of 'mousike' in the Classical Athenian city. Oxford: Oxford University Press, 2004.

NIGHTINGALE, Andrea. Aristotle on the 'Liberal' and 'Illiberal' Arts. Proceedings of the Boston Area Colloquium of Ancient Philosophy, v. 12, n. 1, p. 29-58, 1996.

NUSSBAUM, Martha. Upheavals of thought. The intelligence of emotions. Cambridge: Cambridge University Press, 2001.

ROCCONI, Eleonora. Sounds of war, sounds of peace: for an ethnographic survey of ancient Greek music in Platonic writings. In HICHMANN, E.; EICHMANN, R. (ed.). Musical perceptions, past and present. On etnographic analogy in Music Archcology. Rahden: Leidorf, 2010, p. 37-45. (Studien zur Musikachäologie, 7).

ROCCONI, Eleonora. Music and dance in Greece and Rome. In DESTRÉE, Pierre; MURRAY, Penelope (ed.). A companion to Ancient Aesthetics. Oxford: Wiley-Blackwell, 2015, p. 81-93.

SCHÜTRUMPF, Eckart. Aristoteles Politik Buch VII-VIII. Berlin: Akademie Verlag, 2005.

SOLMSEM, Friedrich. Leisure and play in Aristotle's Ideal State. Rheinisches Museum für Philologie, v. 107, n. 3, p. 193-220, 1964.

SUÑOL, Viviana. El papel de la kátharsis en el programa aristotélico de educación musical. Su relación con la función lúdica y con la educativa de la mousikée. Sintesis. Revista de Filosofía (ex Intus Legere), v. 1, n. 2, p. 122-137, 2018.

SUÑOL, Viviana. La discusión aristotélica sobre los modos de vida. El contraste entre el bios theoretikós en Ética a Nicómaco X 7-8 y el bios praktikós en Politica VII 3. Tópicos, v. 45, p. 9-47, 2013.

SUÑOL, Viviana. La educación como fundamento del (mejor) régimen político en Aristóteles. Éndoxa: Series Filosóficas, v. 36, n. 2, p. 56-73, 2015.

SUÑOL, Viviana. La educación musical en Aristóteles: su correspondencia con la vida mejor en el mejor régimen. Boletín de Estética, año 13, v. 41, p. 8-37, 2017. 
SUÑOL, Viviana. Las funciones de la mousiké en Politica VIII. In SUÑOL, Viviana; MIRANDA, Raquel (ed.). La educación en la filosofía antigua. Ética, retórica y arte en la formación del ciudadano. Buenos Aires: Miño y Dávila, 2020, p.119-139.

SUÑOL, Viviana. La mejor forma de vida en el régimen político ideal de Aristóteles. Anales del Seminario de Historia de la Filosofía, v. 31, n. 2, p. 297-322, 2014.

WOERTHER, Fréderique. Education par la musique et persuasion rhétorique chez Aristote: l'ethos dans la Rhétorique et dans les Politiques (VIII, 5). In MALHOMME, Florence (ed.). Musica rhetoricans. Paris: Presses de l'Université de Paris-Sorbonne, 2002, p. 21 -36.

WOERTHER, Fréderique. L'ethos aristotelicien. Genèse d' une notion rhétorique. Paris: Vrin, 2007.

WOERTHER, Fréderique. Music and the education of the soul in Plato and Aristotle: homeopathy and the formation of the character. Classical Quarterly, v. 58, n. 1, p. 89-103. 2008. 